\title{
PERTURBED THREE-STEP APPROXIMATION PROCESS WITH ERRORS FOR A GENERALIZED IMPLICIT NONLINEAR QUASIVARIATIONAL INCLUSIONS
}

\author{
M. K. AHMAD AND SALAHUDDIN \\ Received 2 January 2006; Revised 28 May 2006; Accepted 30 May 2006
}

We initiate and develop some new perturbed three-step approximation process with errors for solving generalized implicit nonlinear quasivariational inclusions. Also, the convergence and stability of the iterative sequences with errors generated by the algorithms are presented.

Copyright (c) 2006 Hindawi Publishing Corporation. All rights reserved.

\section{Introduction}

Variational inequality theory has become a rich source of inspiration in the pure and applied mathematics. Variational inequalities not only have stimulated new results dealing with nonlinear partial differential equations, but also have been used in a large variety of problems arising in mechanics, physics, optimization and control nonlinear programming, economics and transportation equilibrium and engineering sciences, and so forth. In recent years variational inequalities have been generalized and applied in various directions. For details we refer to $[2,5,6,20]$.

Recently Huang $[10,11]$ constructed some new perturbed Ishikawa and Mann iterative algorithms to approximate the solution of some generalized implicit quasivariational inclusions (inequalities), which includes many iterative algorithms for variational and quasivariational inequality problems as special cases.

On the other hand, Xu [19] revised the definition of Ishikawa and Mann iterative processes with errors and studied the convergence problem of Ishikawa and Mann iterative processes with errors for approximating the solutions of the generative strongly accretive operator equations.

Inspired and motivated by recent research works $[4,7,12,14-16]$, in this paper we initiate and construct some perturbed three-step approximation processes with errors for solving a class of generalized implicit nonlinear quasivariational inclusions. We also discuss the convergence and stability of the iterative sequences generated by algorithms.

Hindawi Publishing Corporation International Journal of Mathematics and Mathematical Sciences Volume 2006, Article ID 43818, Pages 1-15

DOI 10.1155/IJMMS/2006/43818 
2 Perturbed three-step approximation process with errors

\section{Preliminaries}

Let $H$ be a real Hilbert space endowed with a norm $\|\cdot\|$ and an inner product $\langle\cdot, \cdot\rangle$, respectively. For a given maximal monotone mapping $A(\cdot, \cdot): H \times H \rightarrow 2^{H}$ with respect to the first argument, a nonlinear mapping $N(\cdot, \cdot): H \times H \rightarrow H$, three single-valued mappings, $V, G, g: H \rightarrow H$, find $u \in H$ such that $g(u) \in \operatorname{dom} A(\cdot, u)$ and

$$
0 \in g(u)-N(V u, G u)+A(g(u), u)
$$

where $2^{H}$ denote the power subsets of $H$. This variational inclusions is called the generalized implicit nonlinear quasivariational inclusions.

Remark 2.1. (1) We note that $A(g(u), u) \equiv A(g(u))$ for all $u \in H$, then the problem $(2.1)$ is equivalent to finding $u \in H$ such that

$$
0 \in g(u)-N(V u, G u)+A(g(u))
$$

is called general implicit nonlinear quasivariational inclusions.

(2) If $V$ and $G$ are identity mappings, then (2.2) is equivalent to finding $u \in H$ such that

$$
0 \in g(u)-N(u, u)+A(g(u))
$$

(3) We note that $N(u, u) \equiv 0$, zero mapping, then (2.3) is equivalent to the finding $u \in H$ such that $g(u) \in \operatorname{dom} A$,

$$
0 \in g(u)+A(g(u))
$$

is called general variational inclusions considered by Huang et al. [11].

(4) We again note that $g \equiv I$, an identity mapping, then (2.4) is equivalent to the classical variational inclusions, for finding $u \in H$ such that

$$
0 \in u+A(u)
$$

(5) If $A(\cdot, u) \equiv \partial \varphi(\cdot, u)$ is the subdifferential of $\varphi(\cdot, u)$, where $\varphi(\cdot, u): H \times H \rightarrow R \cup$ $\{+\infty\}$ is a proper convex lower semicontinuous functional with respect to the first argument, then the problem $(2.1)$ is equivalent to finding $u \in H$ such that $g(u) \in \operatorname{dom} \partial \varphi(\cdot, u)$ and

$$
\langle g(u)-N(V u, G u), v-g(u)\rangle \geq \varphi(g(u), u)-\varphi(v, u), \quad \forall v \in H
$$

is called generalized nonlinear quasivariational inclusion problems, which is the variant form of Ahmad et al. [1]. 
(6) If $N(V u, G u)=V(u)-G u$, then (2.6) is equivalent to finding $u \in H$ such that $g(u) \in \operatorname{dom} \partial \varphi(\cdot, u)$,

$$
\langle g(u)-(V u-G u), v-g(u)\rangle \geq \varphi(g(u), u)-\varphi(v, u), \quad \forall v \in H,
$$

is called general strongly nonlinear quasivariational inclusions.

(7) If $\partial \varphi(\cdot, u)=\partial \varphi(u)$, then (2.7) is equivalent to finding $u \in H$ such that $g(u) \in$ $\operatorname{dom} A$ and

$$
\langle g(u)-N(V u-G u), v-g(u)\rangle \geq \varphi(g(u))-\varphi(v), \quad \forall v \in H,
$$

is called a variant form of general strongly nonlinear quasivariational inclusions, which is the variant form of that of Khan et al. [13].

(8) If $V$ and $G$ are identity mappings, then (2.6) is equivalent to finding $u \in H$ such that $g(u) \in \operatorname{dom} \partial \varphi(\cdot, u)$,

$$
\langle g(u)-N(u, u), v-g(u)\rangle \geq \varphi(g(u), u)-\varphi(v, u), \quad \forall v \in H,
$$

is called generalized strongly nonlinear implicit quasivariational inclusions.

(9) If $g(u)-N(u, u) \equiv T(u)-A(u)$ and $\varphi(\cdot, u)=\varphi(u)$, then (2.9) reduces to the following problem of finding $u \in H$ such that $g(u) \in \operatorname{dom} \partial \varphi$ and

$$
\langle T u-A u, v-g(u)\rangle \geq \varphi(g(u))-\varphi(v), \quad \forall v \in H,
$$

which is considered by Hassouni and Moudafi [9].

(10) If $K$ is a given closed convex subset of $H$ and $\varphi=I_{K}$ is the indicator function of $K$, defined by

$$
I_{K}(x)= \begin{cases}0, & x \in K, \\ +\infty, & \text { otherwise, }\end{cases}
$$

then, problem (2.3) reduces to the following problem of finding $u \in H$ such that

$$
\langle g(u)-N(V u, G u), v-g(u)\rangle \geq 0, \quad \forall v \in H,
$$

which is variant form of that of Verma [17].

(11) If $V$ and $G$ are identity mappings, then (2.12) is equivalent to finding $u \in H$ such that

$$
\langle g(u)-N(u, u), v-g(u)\rangle \geq 0, \quad \forall v \in H .
$$

(12) If $g(u)-N(u, u)=u-N(u, u)$, then (2.13) is equivalent to finding $u \in H$ such that

$$
\langle u-N(u, u), v-g(u)\rangle \geq 0, \quad \forall v \in H,
$$

is called generalized strongly nonlinear implicit quasivariational inequality problem, considered by Cho et al. [3] . 
4 Perturbed three-step approximation process with errors

(13) If $g \equiv I$ identity mapping, $N(u, u)=T(u)$ for all $u \in H$, and $T: H \rightarrow H$ is a single valued mapping, then (2.14) is equivalent to finding $u \in H$ such that

$$
\langle u-T u, v-u\rangle \geq 0, \quad \forall v \in H,
$$

which is nonlinear variational inequality, considered by Verma [18].

(14) If $u-N(u, u)=T(u)$, then (2.13) reduces to finding $u \in H$ such that

$$
\langle T u, v-g(u)\rangle \geq 0, \quad \forall v \in H,
$$

which is another classical variational inequality introduced by Hartman and Stampacchia $[8]$.

(15) If $g \equiv I$, then (2.15) reduces to finding $u \in H$ such that

$$
\langle T u, v-u\rangle \geq 0, \quad \forall v \in H
$$

which is called classical variational inequality, considered by Hartman and Stampacchia [8].

Definition 2.2. A mapping $g: H \rightarrow H$ is said to be $\alpha$-strongly monotone if there exists a constant $\alpha>0$ such that

$$
\langle g(u)-g(v), u-v\rangle \geq \alpha\|u-v\|^{2}, \quad \forall u, v \in H .
$$

Definition 2.3. A mapping $g: H \rightarrow H$ is said to be $\beta$-Lipschitz continuous if there exists a constant $\beta>0$ such that

$$
\|g(u)-g(v)\| \leq \beta\|u-v\|, \quad \forall u, v \in H .
$$

Definition 2.4. Let $V: H \rightarrow H$ and $N: H \times H \rightarrow H$ be the mappings, $N$ is said to be the following.

(i) $\sigma$-Lipschitz continuous in the first argument if there exists a constant $\sigma>0$ such that

$$
\|N(u, w)-N(v, w)\| \leq \sigma\|u-v\|, \quad \forall u, v, w \in H .
$$

Similarly the Lipschitz continuity of $N$ can be defined with respect to the second argument.

(ii) $\eta$-relaxed monotone with respect to $V$ in the first argument if there exists a constant $\eta>0$ such that

$$
\langle N(V u, w)-N(V v, w), u-v\rangle \geq-\eta\|u-v\|^{2}, \quad \forall u, v, w \in H .
$$

Definition 2.5. Let $G: H \rightarrow H$ and $N: H \times H \rightarrow H$ be the mappings. The mapping $N$ is said to be $\kappa$-relaxed Lipschitz continuous with respect to $G$ in the second argument if there exists a constant $\kappa \geq 0$ such that

$$
\langle N(w, G u)-N(w, G v), u-v\rangle \leq-\kappa\|u-v\|^{2}, \quad \forall u, v, w \in H .
$$


Definition 2.6. Let $H$ be a Hilbert space and let $A: H \rightarrow 2^{H}$ be a maximal monotone mapping. For any fixed $\lambda>0$, the mapping $J_{\lambda}^{A}: H \rightarrow H$ defined by

$$
J_{\lambda}^{A}(u)=(I+\lambda A)^{-1}(u), \quad \forall u \in H
$$

is said to be the resolvent operator of $A$, where $I$ is an identity mapping on $H$.

Lemma 2.7. Let $A: H \rightarrow 2^{H}$ be a maximal monotone mapping. Then the resolvent operator $J_{\lambda}^{A}: H \rightarrow H$ is nonexpansive, that is,

$$
\left\|J_{\lambda}^{A}(u)-J_{\lambda}^{A}(v)\right\| \leq\|u-v\|, \quad \forall u, v \in H
$$

Definition 2.8. Let $\left\{A^{n}\right\}$ and $A$ be the maximal monotone mappings from $H$ into the power set of $H$ for $n=0,1,2, \ldots$. The sequence $\left\{A^{n}\right\}$ is said to be graph-convergence of $A$ (write $A^{n} \stackrel{G}{\longrightarrow} A$ ) if the following property holds.

For every $(u, v) \in \operatorname{Graph}(A)$, there exists a sequence $\left(u_{n}, v_{n}\right) \in \operatorname{Graph}\left(A^{n}\right)$ such that $u_{n} \rightarrow u$ and $v_{n} \rightarrow v$ as $n \rightarrow \infty$.

LEMma 2.9 [2]. Let $\left\{A^{n}\right\}$ and $A$ be the maximal monotone mappings from $H$ into the power set of $H$ for $n=0,1,2, \ldots$. Then $A^{n} \stackrel{G}{\longrightarrow} A$ if and only if

$$
J_{\lambda}^{A^{n}}(u) \rightarrow J_{\lambda}^{A}(u)
$$

for every $u \in H$ and $\lambda>0$.

Lemma 2.10 [15]. Let $\left\{a_{n}\right\},\left\{b_{n}\right\}$, and $\left\{c_{n}\right\}$ be three sequences of nonnegative numbers satisfying the following conditions: there exists a positive integer $n_{0}$ such that

$$
a_{n+1} \leq\left(1-t_{n}\right) a_{n}+b_{n} t_{n}+c_{n}, \quad n \geq n_{0}
$$

where

$$
t_{n} \in[0,1], \quad \sum_{n=0}^{\infty} t_{n}=+\infty, \quad \lim _{n \rightarrow \infty} b_{n}=0, \quad \sum_{n=0}^{\infty} c_{n}<+\infty .
$$

Then $\lim _{n \rightarrow \infty} a_{n}=0$.

LEMmA 2.11. For a given $u \in H, u$ is a solution of the problem (2.1) if and only if

$$
g(u) \equiv J_{\lambda}^{A(\cdot, u)}[g(u)-\lambda(g(u)-N(V u, G u))]
$$

where $\lambda>0$, a constant, $J_{\lambda}^{A(\cdot, u)}(w) \equiv(I+\lambda A(\cdot, u))^{-1}(w)$, where $I$ is an identity operator, and $w \in H$. 
6 Perturbed three-step approximation process with errors

Proof. Let $u \in H$ be a solution of problem (2.1) if and only if for given $\lambda>0$, a constant,

$$
\begin{aligned}
0 & \in g(u)-N(V u, G u)+A(g(u), u) \\
& \Longleftrightarrow 0 \in \lambda g(u)-\lambda N(V u, G u)+\lambda A(g(u), u) \\
& \Longleftrightarrow 0 \in-(g(u)-\lambda(g(u)-N(V u, G u)))+(I+\lambda A(\cdot, u)) g(u) \\
& \Longleftrightarrow g(u)-\lambda(g(u)-N(V u, G u)) \in(I+\lambda A(\cdot, u)) g(u) \\
& \Longleftrightarrow g(u) \equiv(I+\lambda A(\cdot, u))^{-1}[g(u)-\lambda(g(u)-N(V u, G u))] \\
& \Longleftrightarrow g(u) \equiv J_{\lambda}^{A(\cdot, u)}[g(u)-\lambda(g(u)-N(V u, G u))],
\end{aligned}
$$

which completes the proof.

\section{The main results}

Theorem 3.1. Let $g: H \rightarrow H$ be the $\alpha$-strongly monotone and $\beta$-Lipschitz continuous mappings with constants $\alpha>0$ and $\beta>0$, respectively. Let $V, G: H \rightarrow H$ be the two $\xi$-Lipschitz and $\mu$-Lipschitz continuous mappings with constants $\xi>0$ and $\mu>0$, respectively. Let bimapping $N: H \times H \rightarrow H$ be the $\sigma$-Lipschitz continuous with respect to the first argument with constant $\sigma>0$ and $\delta$-Lipschitz continuous with respect to the second argument with constant $\delta>0$. Let $N$ be the $\eta$-relaxed monotone with respect to $V$ in the first argument with constant $\eta>0$ and $\kappa$-relaxed Lipschitz continuous with respect to $G$ in the second argument with constant $\kappa \geq 0$. Let $A: H \times H \rightarrow 2^{H}$ be the maximal monotone with respect to the first argument. It assume that for all $u, v, w \in H$,

$$
\left\|J_{\lambda}^{A(\cdot, u)}(w)-J_{\lambda}^{A(\cdot, v)}(w)\right\| \leq \rho\|u-v\|,
$$

where $\rho>0$ is a constant. If

$$
\begin{gathered}
\left|\lambda-\frac{\eta-p(1-k)}{\sigma^{2} \xi^{2}-p^{2}}\right|<\frac{\sqrt{(\eta-p(1-k))^{2}-\left(\sigma^{2} \xi^{2}-p^{2}\right) k(2-k)}}{\sigma^{2} \xi^{2}-p^{2}}, \\
\quad \eta>p(1-k)+\sqrt{\left(\sigma^{2} \xi^{2}-p^{2}\right) k(2-k)}, \quad k<1, p<\sigma \xi, \eta>p
\end{gathered}
$$

where

$$
\begin{gathered}
\theta=k+\sqrt{1-2 \lambda \eta+\lambda^{2} \sigma^{2} \xi^{2}}+\lambda p<1, \\
k=2 \Omega+\rho, \quad p=\Omega+\omega, \quad \Omega=\sqrt{1-2 \alpha+\beta^{2}}, \quad \omega=\sqrt{1+2 \kappa+\delta^{2} \mu^{2}} .
\end{gathered}
$$

Then the problem (2.1) has a unique solution $u \in H$.

Proof. Define a mapping $F: H \rightarrow H$ as

$$
F(u)=u-g(u)+J_{\lambda}^{A(\cdot, u)}[g(u)-\lambda(g(u)-N(V u, G u))], \quad u \in H .
$$


By Lemmas 2.11 and 2.7, it is enough to show that $F$ is a contraction mapping. It follows from (3.4) that

$$
\begin{aligned}
\|F(u)-F(v)\|= & \|u-v-(g(u)-g(v))\| \\
& +\| J_{\lambda}^{A(\cdot, u)}[g(u)-\lambda(g(u)-N(V u, G u))] \\
& \quad-J_{\lambda}^{A(\cdot, u)}[g(v)-\lambda(g(v)-N(V v, G v))] \| \\
& +\| J_{\lambda}^{A(\cdot, u)}[g(v)-\lambda(g(v)-N(V v, G v))] \\
& \quad-J_{\lambda}^{A(\cdot, v)}[g(v)-\lambda(g(v)-N(V v, G v))] \| \\
& +\|g(u)-g(v)-\lambda(g(u)-N(V u, G u))+\lambda(g(v)-N(V v, G v))\| \\
& +\rho\|u-v\| \\
\leq & 2\|u-v-(g(u)-g(v))\| \\
& +\|u-v+\lambda(N(V u, G u)-N(V v, G u))\| \\
& +\lambda\|g(u)-g(v)-(N(V v, G u)-N(V v, G v))\| \\
& +\rho\|u-v\| \\
\leq & 2\|u-v-(g(u)-g(v))\| \\
& +\|u-v+\lambda(N(V u, G u)-N(V v, G u))\| \\
& +\lambda\|u-v-(g(u)-g(v))\| \\
& +\lambda\|u-v-(N(V v, G u)-N(V v, G v))\|+\rho\|u-v\| .
\end{aligned}
$$

Since $g$ is $\alpha$-strongly monotone and $\beta$-Lipschitz continuous, we have

$$
\|u-v-(g(u)-g(v))\|^{2} \leq\left(1-2 \alpha+\beta^{2}\right)\|u-v\|^{2} .
$$

Since $N$ is $\sigma$-Lipschitz continuous with respect to first argument, $V$ is $\xi$-Lipschitz continuous, and $N$ is $\eta$-relaxed monotone with respect to $V$ in the first argument with constant $\eta>0$, we have

$$
\begin{aligned}
\| u-v & +\lambda(N(V u, G u)-N(V v, G u)) \|^{2} \\
\leq & \|u-v\|^{2}+2 \lambda\langle N(V u, G u)-N(V v, G u), u-v\rangle \\
& +\lambda^{2}\|N(V u, G u)-N(V v, G u)\|^{2} \\
\leq & \|u-v\|^{2}-2 \lambda \eta\|u-v\|^{2}+\lambda^{2} \sigma^{2} \xi^{2}\|u-v\|^{2} \\
\leq & \left(1-2 \lambda \eta+\lambda^{2} \sigma^{2} \xi^{2}\right)\|u-v\|^{2} .
\end{aligned}
$$

Since $N$ is $\delta$-Lipschitz continuous with respect to the second argument, $G$ is $\mu$-Lipschitz continuous, and $N$ is $\kappa$-relaxed Lipschitz continuous with respect to $G$ in the second 
8 Perturbed three-step approximation process with errors

argument with constant $\kappa \geq 0$, we get

$$
\begin{aligned}
\| u-v- & (N(V v, G u)-N(V v, G u)) \|^{2} \\
\leq & \|u-v\|^{2}-2\langle N(V v, G u)-N(V v, G v), u-v\rangle \\
& +\|N(V v, G u)-N(V v, G v)\|^{2} \\
\leq & \|u-v\|^{2}+2 \kappa\|u-v\|^{2}+\delta^{2} \mu^{2}\|u-v\|^{2} \\
\leq & \left(1+2 \kappa+\delta^{2} \mu^{2}\right)\|u-v\|^{2} .
\end{aligned}
$$

It follows from (3.5)-(3.8) that we have

$$
\begin{aligned}
\| F(u)- & F(v) \| \\
\leq & 2 \sqrt{1-2 \alpha+\beta^{2}}\|u-v\|+\sqrt{1-2 \lambda \eta+\lambda^{2} \sigma^{2} \xi^{2}}\|u-v\| \\
& +\lambda \sqrt{1-2 \alpha+\beta^{2}}\|u-v\|+\lambda \sqrt{1+2 \kappa+\delta^{2} \mu^{2}}\|u-v\|+\rho\|u-v\| \\
\leq & {\left[2 \sqrt{1-2 \alpha+\beta^{2}}+\rho+\sqrt{1-2 \lambda \eta+\lambda^{2} \sigma^{2} \xi^{2}}+\lambda \sqrt{1-2 \alpha+\beta^{2}}\right.} \\
& \left.+\lambda \sqrt{1+2 \kappa+\delta^{2} \mu^{2}}\right]\|u-v\| \\
\leq & {\left[2 \Omega+\rho+\sqrt{1-2 \lambda \eta+\lambda^{2} \sigma^{2} \xi^{2}}+\lambda \Omega+\lambda \omega\right]\|u-v\| } \\
\leq & {\left[k+\sqrt{1-2 \lambda \eta+\lambda^{2} \sigma^{2} \xi^{2}}+\lambda(\Omega+\omega)\right]\|u-v\| } \\
\leq & {\left[k+\sqrt{1-2 \lambda \eta+\lambda^{2} \sigma^{2} \xi^{2}}+\lambda p\right]\|u-v\| } \\
\leq & \theta\|u-v\|,
\end{aligned}
$$

where

$$
\begin{gathered}
\theta=k+\sqrt{1-2 \lambda \eta+\lambda^{2} \sigma^{2} \xi^{2}}+\lambda p, \\
k=2 \Omega+\rho, \quad p=\Omega+\omega, \quad \Omega=\sqrt{1-2 \alpha+\beta^{2}}, \quad \omega=\sqrt{1+2 \kappa+\delta^{2} \mu^{2}} .
\end{gathered}
$$

It is easy to verify that $(3.3)$ means $0<\theta<1$. Hence $F$ is a contraction mapping and has a fixed point $u \in H$. It follows from Lemma 2.11 that $u$ is a unique solution of problem (2.1). This completes the proof.

Now we suggest the following perturbed iterative approximation process with errors for solving (2.1). 
Algorithm 3.2. For any given $u_{0} \in H$, compute the approximate solution $\left\{u_{n}\right\}$ by the perturbed iterative process with errors:

$$
\begin{gathered}
u_{n+1}=\left(1-\alpha_{n}\right) u_{n}+\alpha_{n}\left[v_{n}-g\left(v_{n}\right)+J_{\lambda}^{A_{n}\left(\cdot, v_{n}\right)}\left\{g\left(v_{n}\right)-\lambda\left(g\left(v_{n}\right)-N\left(V v_{n}, G v_{n}\right)\right)\right\}\right]+\alpha_{n} e_{n}+l_{n}, \\
v_{n}=\left(1-\beta_{n}\right) u_{n}+\beta_{n}\left[w_{n}-g\left(w_{n}\right)+J_{\lambda}^{A_{n}\left(\cdot, w_{n}\right)}\left\{g\left(w_{n}\right)-\lambda\left(g\left(w_{n}\right)-N\left(V w_{n}, G w_{n}\right)\right)\right\}\right]+f_{n}, \\
w_{n}=\left(1-\gamma_{n}\right) u_{n}+\gamma_{n}\left[u_{n}-g\left(u_{n}\right)+J_{\lambda}^{A_{n}\left(\cdot, u_{n}\right)}\left\{g\left(u_{n}\right)-\lambda\left(g\left(u_{n}\right)-N\left(V u_{n}, G u_{n}\right)\right)\right\}\right]+h_{n},
\end{gathered}
$$

for all $n \geq 0$, where $\left\{\alpha_{n}\right\},\left\{\beta_{n}\right\}$, and $\left\{\gamma_{n}\right\}$ are sequences in $[0,1]$; and $\left\{e_{n}\right\},\left\{f_{n}\right\},\left\{l_{n}\right\}$, and $\left\{h_{n}\right\}$ are bounded sequences in $H$, satisfying suitable conditions:

$$
\sum_{n=0}^{n} \alpha_{n}=+\infty, \quad \sum_{n=0}^{n}\left\|l_{n}\right\|<+\infty, \quad \lim _{n \rightarrow \infty}\left\|e_{n}\right\|=\lim _{n \rightarrow \infty}\left\|f_{n}\right\|=\lim _{n \rightarrow \infty}\left\|h_{n}\right\|=0,
$$

and $\lambda>0$ is a constant.

If we remark that $\gamma_{n}=0$ and $h_{n}=0$, for $n \geq 0$, then Algorithm 3.2 reduces to the following.

Algorithm 3.3. For any given $u_{0} \in H$, compute the approximate solution $\left\{u_{n}\right\}$ by the perturbed Ishikawa iterative process with errors:

$$
\begin{gathered}
u_{n+1}=\left(1-\alpha_{n}\right) u_{n}+\alpha_{n}\left[v_{n}-g\left(v_{n}\right)+J_{\lambda}^{A_{n}\left(\cdot, v_{n}\right)}\left\{g\left(v_{n}\right)-\lambda\left(g\left(v_{n}\right)-N\left(V v_{n}, G v_{n}\right)\right)\right\}\right]+e_{n} \alpha_{n}+l_{n}, \\
v_{n}=\left(1-\beta_{n}\right) u_{n}+\beta_{n}\left[u_{n}-g\left(u_{n}\right)+J_{\lambda}^{A_{n}\left(\cdot, u_{n}\right)}\left\{g\left(u_{n}\right)-\lambda\left(g\left(u_{n}\right)-N\left(V u_{n}, G u_{n}\right)\right)\right\}\right]+f_{n},
\end{gathered}
$$

where $\left\{\alpha_{n}\right\},\left\{\beta_{n}\right\},\left\{e_{n}\right\},\left\{f_{n}\right\}$, and $\left\{l_{n}\right\}$ are the same as in Algorithm 3.2.

If $\beta_{n}=0$ and $f_{n}=0$ for $n \geq 0$, then Algorithm 3.3 reduces to the following.

Algorithm 3.4. For any given $u_{0} \in H$, compute the approximate solution $\left\{u_{n}\right\}$ by the perturbed Mann iterative process with errors:

$$
\begin{aligned}
u_{n+1}= & \left(1-\alpha_{n}\right) u_{n}+\alpha_{n}\left[u_{n}-g\left(u_{n}\right)+J_{\lambda}^{A_{n}\left(\cdot, u_{n}\right)}\left\{g\left(u_{n}\right)-\lambda\left(g\left(u_{n}\right)-N\left(V u_{n}, G u_{n}\right)\right)\right\}\right] \\
& +e_{n} \alpha_{n}+l_{n},
\end{aligned}
$$

where $\left\{\alpha_{n}\right\},\left\{e_{n}\right\}$, and $\left\{l_{n}\right\}$ are the same as in Algorithm 3.2.

If $e_{n}=f_{n}=h_{n}=l_{n}=0, n \geq 0$, then Algorithm 3.2 reduces to the following. 
Algorithm 3.5. For any given $u_{0} \in H$, compute the approximate solution $\left\{u_{n}\right\}$ by the iterative process:

$$
\begin{gathered}
u_{n+1}=\left(1-\alpha_{n}\right) u_{n}+\alpha_{n}\left[v_{n}-g\left(v_{n}\right)+J_{\lambda}^{A_{n}\left(\cdot, v_{n}\right)}\left\{g\left(v_{n}\right)-\lambda\left(g\left(v_{n}\right)-N\left(V v_{n}, G v_{n}\right)\right)\right\}\right], \\
v_{n}=\left(1-\beta_{n}\right) u_{n}+\beta_{n}\left[w_{n}-g\left(w_{n}\right)+J_{\lambda}^{A_{n}\left(\cdot, w_{n}\right)}\left\{g\left(w_{n}\right)-\lambda\left(g\left(w_{n}\right)-N\left(V w_{n}, G w_{n}\right)\right)\right\}\right], \\
w_{n}=\left(1-\gamma_{n}\right) u_{n}+\gamma_{n}\left[u_{n}-g\left(u_{n}\right)+J_{\lambda}^{A_{n}\left(\cdot, u_{n}\right)}\left\{g\left(u_{n}\right)-\lambda\left(g\left(u_{n}\right)-N\left(V u_{n}, G u_{n}\right)\right)\right\}\right],
\end{gathered}
$$

where $\alpha_{n}, \beta_{n}$, and $\gamma_{n}$ are the same as in Algorithm 3.2.

Now we discuss the convergence and stability of the iterative sequences with errors generated by Algorithm 3.2; we first give some concepts.

Let $T$ be a self map of $H, x_{0} \in H$, and $x_{n+1}=f\left(T, x_{n}\right)$, define an iterative procedure which yields a sequence of points $\left\{x_{n}\right\}$ in $H$. Suppose that $\{x \in H: T x=x\}=\varnothing$ and $\left\{x_{n}\right\}$ converge to a fixed point $x^{\star} \in H$. Let $\left\{y_{n}\right\} \subset H$ and $\epsilon_{n}=\left\|y_{n+1}-f\left(T, y_{n}\right)\right\|$. If $\lim _{n \rightarrow \infty} \epsilon_{n}=0$ implies that $\lim _{n \rightarrow \infty} y_{n}=x^{\star}$, then the iterative procedure $\left\{x_{n}\right\}$ defined by $x_{n+1}=f\left(T, x_{n}\right)$ is said to be $T$-stable or stable with respect to $T$. If $\sum_{n=0}^{\infty} \epsilon_{n}<+\infty$ implies that $\lim _{n \rightarrow \infty} y_{n}=x^{\star}$, then the iterative procedure $\left\{x_{n}\right\}$ is said to almost $T$-stable.

Remark 3.6. An iterative procedure $\left\{x_{n}\right\}$ which is $T$-stable is almost $T$-stable, and an iterative procedure $\left\{x_{n}\right\}$ which is almost $T$-stable need not be $T$-stable, see [5].

Theorem 3.7. Let $g, V, G$, and $N$ be the same as in Theorem 3.1, and the conditions (3.1) and (3.3) hold. Let $\left\{A_{n}\right\}$ and $A$ be maximal monotone mappings from $H$ into power set of $H$ such that $A_{n} \stackrel{G}{\longrightarrow}$ A. Let $\left\{x_{n}\right\}$ be a sequence in $H$ and define a sequence $\left\{\epsilon_{n}\right\}$ of real numbers as follows:

$$
\begin{aligned}
& \epsilon_{n}=\| x_{n+1}=\left\{\left(1-\alpha_{n}\right) x_{n}\right. \\
& +\alpha_{n}\left[y_{n}-g\left(y_{n}\right)+J_{\lambda}^{A_{n}\left(\cdot, y_{n}\right)}\left(g\left(y_{n}\right)-\lambda\left(g\left(y_{n}\right)-N\left(V y_{n}, G y_{n}\right)\right)\right)\right] \\
& \left.+\alpha_{n} e_{n}+l_{n}\right\} \|, \\
& y_{n}=\left(1-\beta_{n}\right) x_{n}+\beta_{n}\left[z_{n}-g\left(z_{n}\right)+J_{\lambda}^{A_{n}\left(\cdot, z_{n}\right)}\left(g\left(z_{n}\right)-\lambda\left(g\left(z_{n}\right)-N\left(V z_{n}, G z_{n}\right)\right)\right)\right]+f_{n}, \\
& z_{n}=\left(1-\gamma_{n}\right) x_{n}+\gamma_{n}\left[x_{n}-g\left(x_{n}\right)+J_{\lambda}^{A_{n}\left(\cdot, x_{n}\right)}\left(g\left(x_{n}\right)-\lambda\left(g\left(x_{n}\right)-N\left(V x_{n}, G x_{n}\right)\right)\right)\right]+h_{n} .
\end{aligned}
$$

Then the following conditions hold.

(i) The sequence $\left\{u_{n}\right\}$ generated by Algorithm 3.2 converges strongly to the unique solution $u^{\star}$ of the problem (2.1).

(ii) If $\epsilon_{n}=\alpha_{n} D_{n}+q_{n}$ with $\sum_{n=0}^{\infty} q_{n}<+\infty$ and $\lim _{n \rightarrow \infty} D_{n}=0$, then

$$
\lim _{n \rightarrow \infty} x_{n}=u^{\star} .
$$

(iii) $\lim _{n \rightarrow \infty} x_{n}=u^{\star}$ implies that

$$
\lim _{n \rightarrow \infty} \epsilon_{n}=0 .
$$


Proof. By Theorem 3.1, we know that problem (2.1) has a unique solution $u^{\star} \in H$. It is easy to see that conclusion (i) follows from (ii). Now we prove (ii). It follows from the Lemma 2.11 that

$$
\begin{aligned}
u^{\star} & =\left(1-\alpha_{n}\right) u^{\star}+\alpha_{n}\left[u^{\star}-g\left(u^{\star}\right)+J_{\lambda}^{A\left(\cdot, u^{\star}\right)}\left\{g\left(u^{\star}\right)-\lambda\left(g\left(u^{\star}\right)-N\left(V u^{\star}, G u^{\star}\right)\right)\right\}\right] \\
& =\left(1-\beta_{n}\right) u^{\star}+\beta_{n}\left[u^{\star}-g\left(u^{\star}\right)+J_{\lambda}^{A\left(\cdot, u^{\star}\right)}\left\{g\left(u^{\star}\right)-\lambda\left(g\left(u^{\star}\right)-N\left(V u^{\star}, G u^{\star}\right)\right)\right\}\right] \\
& =\left(1-\gamma_{n}\right) u^{\star}+\gamma_{n}\left[u^{\star}-g\left(u^{\star}\right)+J_{\lambda}^{A\left(\cdot, u^{\star}\right)}\left\{g\left(u^{\star}\right)-\lambda\left(g\left(u^{\star}\right)-N\left(V u^{\star}, G u^{\star}\right)\right)\right\}\right] .
\end{aligned}
$$

From (3.16) and (3.19), we have

$$
\begin{aligned}
& \left\|x_{n+1}-u^{\star}\right\| \\
& \leq \| x_{n+1}-\left\{\left(1-\alpha_{n}\right) x_{n}\right. \\
& +\alpha_{n}\left[y_{n}-g\left(y_{n}\right)+J_{\lambda}^{A_{n}\left(\cdot, y_{n}\right)}\left(g\left(y_{n}\right)-\lambda\left(g\left(y_{n}\right)-N\left(V y_{n}, G y_{n}\right)\right)\right)\right] \\
& \left.+\alpha_{n} e_{n}+l_{n}\right\} \| \\
& +\|\left(1-\alpha_{n}\right)\left(x_{n}-u^{\star}\right)+\alpha_{n}\left[y_{n}-u^{\star}-\left(g\left(y_{n}\right)-g\left(u^{\star}\right)\right)\right] \\
& +\alpha_{n}\left[J_{\lambda}^{A_{n}\left(\cdot, y_{n}\right)}\left(g\left(y_{n}\right)-\lambda\left(g\left(y_{n}\right)-N\left(V y_{n}, G y_{n}\right)\right)\right)\right. \\
& \left.-J_{\lambda}^{A\left(\cdot, u^{\star}\right)}\left(g\left(u^{\star}\right)-\lambda\left(g\left(u^{\star}\right)-N\left(V u^{\star}, G u^{\star}\right)\right)\right)\right] \| \\
& +\alpha_{n}\left\|e_{n}\right\|+\left\|l_{n}\right\| \\
& \leq\left(1-\alpha_{n}\right)\left\|x_{n}-u^{\star}\right\|+\alpha_{n}\left\|y_{n}-u^{\star}-\left(g\left(y_{n}\right)-g\left(u^{\star}\right)\right)\right\| \\
& +\alpha_{n} \| J_{\lambda}^{A_{n}\left(\cdot, y_{n}\right)}\left(g\left(y_{n}\right)-\lambda\left(g\left(y_{n}\right)-N\left(V y_{n}, G y_{n}\right)\right)\right) \\
& -J_{\lambda}^{A_{n}\left(\cdot, y_{n}\right)}\left(g\left(u^{\star}\right)-\lambda\left(g\left(u^{\star}\right)-N\left(V u^{\star}, G u^{\star}\right)\right)\right) \| \\
& +\alpha_{n} \| J_{\lambda}^{A_{n}\left(\cdot, y_{n}\right)}\left(g\left(u^{\star}\right) \lambda\left(g\left(u^{\star}\right)-N\left(V u^{\star}, G u^{\star}\right)\right)\right) \\
& -J_{\lambda}^{A\left(\cdot, u^{\star}\right)}\left(g\left(u^{\star}\right)-\lambda\left(g\left(u^{\star}\right)-N\left(V u^{\star}, G u^{\star}\right)\right)\right) \| \\
& +\alpha_{n} \| J_{\lambda}^{A_{n}\left(\cdot, u^{\star}\right)}\left(g\left(u^{\star}\right)-\lambda\left(g\left(u^{\star}\right)-N\left(V u^{\star}, G u^{\star}\right)\right)\right) \\
& -J_{\lambda}^{A\left(\cdot, u^{\star}\right)}\left(g\left(u^{\star}\right)-\lambda\left(g\left(u^{\star}\right)-N\left(V u^{\star}, G u^{\star}\right)\right)\right)\left\|+\epsilon_{n}+\alpha_{n}\right\| e_{n}\|+\| l_{n} \| \\
& \leq\left(1-\alpha_{n}\right)\left\|x_{n}-u^{\star}\right\|+2 \alpha_{n}\left\|y_{n}-u^{\star}-\left(g\left(y_{n}\right)-g\left(u^{\star}\right)\right)\right\| \\
& +\alpha_{n}\left\|y_{n}-u^{\star}+\lambda\left(N\left(V y_{n}, G y_{n}\right)-N\left(V u^{\star}, G y_{n}\right)\right)\right\| \\
& +\alpha_{n} \lambda\left\|y_{n}-u^{\star}-\left(g\left(y_{n}\right)-g\left(u^{\star}\right)\right)\right\| \\
& +\alpha_{n} \lambda\left\|y_{n}-u^{\star}-\left(N\left(V u^{\star}, G y_{n}\right)-N\left(V u^{\star}, G u^{\star}\right)\right)\right\| \\
& +\alpha_{n} \rho\left\|y_{n}-u^{\star}\right\|+\epsilon_{n}+\alpha_{n} P_{n}+\alpha_{n}\left\|e_{n}\right\|+\left\|l_{n}\right\| \text {, }
\end{aligned}
$$


12 Perturbed three-step approximation process with errors

where

$$
\begin{aligned}
P_{n}= & \| J_{\lambda}^{A_{n}\left(\cdot, u^{\star}\right)}\left(g\left(u^{\star}\right)-\lambda\left(g\left(u^{\star}\right)-N\left(V u^{\star}, G u^{\star}\right)\right)\right) \\
& \quad-J_{\lambda}^{A\left(\cdot, u^{\star}\right)}\left(g\left(u^{\star}\right)-\lambda\left(g\left(u^{\star}\right)-N\left(V u^{\star}, G u^{\star}\right)\right)\right) \| \longrightarrow 0 .
\end{aligned}
$$

As the proof of (3.6)-(3.8), we have

$$
\begin{gathered}
\left\|y_{n}-u^{\star}-\left(g\left(y_{n}\right)-g\left(u^{\star}\right)\right)\right\| \leq \sqrt{1-2 \alpha+\beta^{2}}\left\|y_{n}-u^{\star}\right\|, \\
\left\|y_{n}-u^{\star}+\lambda\left(N\left(V y_{n}, G y_{n}\right)-N\left(V u^{\star}, G y_{n}\right)\right)\right\| \leq \sqrt{1-2 \lambda \eta+\lambda^{2} \sigma^{2} \xi^{2}}\left\|y_{n}-u^{\star}\right\|, \\
\left\|y_{n}-u^{\star}-\left(N\left(V u^{\star}, G y_{n}\right)-N\left(V u^{\star}, G u^{\star}\right)\right)\right\| \leq \sqrt{1+2 \kappa+\delta^{2} \mu^{2}}\left\|y_{n}-u^{\star}\right\| .
\end{gathered}
$$

It follows from (3.20)-(3.22) that

$$
\left\|x_{n+1}-u^{\star}\right\| \leq\left(1-\alpha_{n}\right)|| x_{n}-u^{\star}||+\theta \alpha_{n}\left\|y_{n}-u^{\star}\right\|+\epsilon_{n}+\alpha_{n} P_{n}+\alpha_{n}\left\|e_{n}\right\|+\left\|l_{n}\right\|,
$$

where

$$
\begin{gathered}
\theta=k+\sqrt{1-2 \lambda \eta+\lambda^{2} \sigma^{2} \xi^{2}}+\lambda p<1, \\
k=2 \Omega+\rho, \quad p=\Omega+\omega, \quad \Omega=\sqrt{1-2 \alpha+\beta^{2}}, \quad \omega=\sqrt{1+2 \kappa+\delta^{2} \mu^{2}} .
\end{gathered}
$$

Similarly, we have

$$
\begin{aligned}
\left\|y_{n}-u^{\star}\right\| & \leq\left(1-\beta_{n}\right)\left\|x_{n}-u^{\star}\right\|+\beta_{n} \theta\left\|z_{n}-u^{\star}\right\|+\beta_{n} P_{n}+\left\|f_{n}\right\|, \\
\left\|z_{n}-u^{\star}\right\| & \leq\left(1-\gamma_{n}\right)\left\|x_{n}-u^{\star}\right\|+\gamma_{n} \theta\left\|x_{n}-u^{\star}\right\|+\gamma_{n} P_{n}+\left\|h_{n}\right\| \\
& \leq\left(1-\gamma_{n}(1-\theta)\right)\left\|x_{n}-u^{\star}\right\|+\gamma_{n} P_{n}+\left\|h_{n}\right\| \\
& \leq\left\|x_{n}-u^{\star}\right\|+\gamma_{n} P_{n}+\left\|h_{n}\right\|,
\end{aligned}
$$

where

$$
\left(1-\gamma_{n}(1-\theta)\right) \leq 1
$$

Substituting (3.26) into (3.25), we have

$$
\begin{aligned}
\left\|y_{n}-u^{\star}\right\| & \leq\left(1-\beta_{n}\right)\left\|x_{n}-u^{\star}\right\|+\beta_{n} \theta\left\|x_{n}-u^{\star}\right\|+\beta_{n} \gamma_{n} P_{n} \theta+\beta_{n} \theta\left\|h_{n}\right\|+\beta_{n} P_{n}+\left\|f_{n}\right\| \\
& \leq\left(1-\beta_{n}(1-\theta)\right)\left\|x_{n}-u^{\star}\right\|+\beta_{n} \gamma_{n} P_{n} \theta+\beta_{n} \theta\left\|h_{n}\right\|+\beta_{n} P_{n}+\left\|f_{n}\right\| \\
& \leq\left\|x_{n}-u^{\star}\right\|+2 P_{n}+\left\|h_{n}\right\|+\left\|f_{n}\right\| .
\end{aligned}
$$


From (3.23) and (3.28),

$$
\begin{aligned}
\left\|x_{n+1}-u^{\star}\right\| \leq & \left(1-\alpha_{n}\right)|| x_{n}-u^{\star}\left\|+\alpha_{n} \theta\right\| x_{n}-u^{\star} \|+\alpha_{n} \theta\left(2 P_{n}+\left\|h_{n}\right\|+\left\|f_{n}\right\|\right) \\
& +\alpha_{n} \Delta_{n}+q_{n}+\alpha_{n} P_{n}+\alpha_{n}\left\|e_{n}\right\|+\left\|l_{n}\right\| \\
\leq & \left(1-\alpha_{n}(1-\theta)\right)\left\|x_{n}-u^{\star}\right\| \\
& +\alpha_{n}(1-\theta) \frac{1}{1-\theta}\left(3 P_{n}+\left\|h_{n}\right\|+\left\|f_{n}\right\|+\Delta_{n}+\left\|e_{n}\right\|\right)+\left(q_{n}+\left\|l_{n}\right\|\right) .
\end{aligned}
$$

Set $a_{n}=x_{n}-u^{\star}, b_{n}=(1 /(1-\theta))\left(3 P_{n}+\left\|h_{n}\right\|+\left\|f_{n}\right\|+\Delta_{n}+\left\|e_{n}\right\|\right)$ and $t_{n}=\alpha_{n}(1-\theta)$, $c_{n}=q_{n}+\left\|l_{n}\right\|$.

Then we can rewrite (3.29) as follows:

$$
a_{n+1} \leq\left(1-t_{n}\right) a_{n}+b_{n} t_{n}+c_{n}
$$

From the assumption,we know that $\left\{a_{n}\right\},\left\{b_{n}\right\},\left\{c_{n}\right\}$, and $\left\{t_{n}\right\}$ satisfy the conditions of Lemma 2.10. It follows from Lemma 2.10 that $a_{n} \rightarrow 0$ and so $x_{n} \rightarrow u^{\star}$ as $n \rightarrow \infty$.

Next, we prove (iii). From (3.11) and (3.28), we know that $y_{n} \rightarrow u^{\star}$ as $n \rightarrow \infty$. It follows from (3.16) that

$$
\begin{aligned}
\epsilon_{n} \leq & \left\|x_{n}-u^{\star}\right\| \\
& +\left\|\left(1-\alpha_{n}\right) x_{n}+\alpha_{n}\left[y_{n}-g\left(y_{n}\right)+J_{\lambda}^{A_{n}\left(\cdot, y_{n}\right)}\left(g\left(y_{n}\right)-\lambda\left(g\left(y_{n}\right)-N\left(V y_{n}, G y_{n}\right)\right)\right)\right]-u^{\star}\right\| \\
& +\alpha_{n}\left\|e_{n}\right\|+\left\|l_{n}\right\| .
\end{aligned}
$$

As the proof of (3.23), we have

$$
\begin{aligned}
& \left\|\left(1-\alpha_{n}\right) x_{n}+\alpha_{n}\left[y_{n}-g\left(y_{n}\right)+J_{\lambda}^{A_{n}\left(\cdot, y_{n}\right)}\left(g\left(y_{n}\right)-\lambda\left(g\left(y_{n}\right)-N\left(V y_{n}, G y_{n}\right)\right)\right)\right]-u^{\star}\right\| \\
& \leq\left(1-\alpha_{n}\right)\left\|x_{n}-u^{\star}\right\|+\alpha_{n} \theta\left\|y_{n}-u^{\star}\right\|+\alpha_{n} P_{n} .
\end{aligned}
$$

Substituting (3.32) and (3.31), we get

$$
\epsilon_{n} \leq\left\|x_{n+1}-u^{\star}\right\|+\left(1-\alpha_{n}\right)\left\|x_{n}-u^{\star}\right\|+\alpha_{n} \theta\left\|y_{n}-u^{\star}\right\|+\alpha_{n} P_{n}
$$

Since $x_{n} \rightarrow u^{\star}, y_{n} \rightarrow u^{\star}$ and $P_{n} \rightarrow 0$ as $n \rightarrow \infty$, it follows that $\epsilon_{n} \rightarrow 0$. This completes the proof. 
14 Perturbed three-step approximation process with errors

\section{Acknowledgment}

The authors are thankful to the anonymous referee for the valuable comments that resulted in the improvement of the manuscript.

\section{References}

[1] R. Ahmad, K. R. Kazmi, and Salahuddin, Completely generalized non-linear variational inclusions involving relaxed Lipschitz and relaxed monotone mappings, Nonlinear Analysis Forum 5 (2000), $61-69$.

[2] H. Attouch, Variational Convergence for Functions and Operators, Math. Ser., Pitman, London, 1974.

[3] Y. J. Cho, J. H. Kim, N. J. Huang, and S. M. Kang, Ishikawa and Mann iterative processes with errors for generalized strongly nonlinear implicit quasi-variational inequalities, Publicationes Mathematicae Debrecen 58 (2001), no. 4, 635-649.

[4] K. Deimling, Zeros of accretive operators, Manuscripta Mathematica 13 (1974), no. 4, 365-374.

[5] F. Giannessi and A. Maugeri (eds.), Variational Inequalities and Network Equilibrium Problems, Plenum Press, New York, 1995.

[6] R. Glowinski, J.-L. Lions, and R. Trémolières, Numerical Analysis of Variational Inequalities, Studies in Mathematics and Its Applications, vol. 8, North-Holland, Amsterdam, 1981.

[7] A. M. Harder and T. L. Hicks, Stability results for fixed point iteration procedures, Mathematica Japonica 33 (1988), no. 5, 693-706.

[8] P. Hartman and G. Stampacchia, On some non-linear elliptic differential-functional equations, Acta Mathematica 115 (1966), no. 1, 271-310.

[9] A. Hassouni and A. Moudafi, A perturbed algorithm for variational inclusions, Journal of Mathematical Analysis and Applications 185 (1994), no. 3, 706-712.

[10] N.-J. Huang, Mann and Ishikawa type perturbed iterative algorithms for generalized nonlinear implicit quasi-variational inclusions, Computers \& Mathematics with Applications 35 (1998), no. 10, $1-7$.

[11] N.-J. Huang, Y.-P. Fang, and Y. J. Cho, Perturbed three-step approximation processes with errors for a class of general implicit variational inclusions, Journal of Nonlinear and Convex Analysis 4 (2003), no. 2, 301-308.

[12] S. Ishikawa, Fixed points by a new iteration method, Proceedings of the American Mathematical Society 44 (1974), no. 1, 147-150.

[13] M. F. Khan, R. Ahmad, and Q. H. Ansari, Generalized multivalued nonlinear variational inclusions with relaxed Lipschitz and relaxed monotone mappings, Advances in Nonlinear Variational Inequalities 3 (2000), no. 1, 93-101.

[14] Q. Liu, Convergence theorems of the sequence of iterates for asymptotically demicontractive and hemicontractive mappings, Nonlinear Analysis. Theory, Methods \& Applications 26 (1996), no. 11, 1835-1842.

[15] W. R. Mann, Mean value methods in iteration, Proceedings of the American Mathematical Society 4 (1953), no. 3, 506-510.

[16] M. A. Noor, New approximation schemes for general variational inequalities, Journal of Mathematical Analysis and Applications 251 (2000), no. 1, 217-229.

[17] R. U. Verma, On generalized variational inequalities involving relaxed Lipschitz and relaxed monotone operators, Journal of Mathematical Analysis and Applications 213 (1997), no. 1, 387-392.

[18] Generalized pseudo-contractions and nonlinear variational inequalities, Publicationes Mathematicae Debrecen 53 (1998), no. 1-2, 23-28. 
[19] Y. Xu, Ishikawa and Mann iterative processes with errors for nonlinear strongly accretive operator equations, Journal of Mathematical Analysis and Applications 224 (1998), no. 1, 91-101.

[20] G. X.-Z. Yuan, KKM Theory and Applications in Nonlinear Analysis, Monographs and Textbooks in Pure and Applied Mathematics, vol. 218, Marcel Dekker, New York, 1999.

M. K. Ahmad: Department of Mathematics, Aligarh Muslim University, Aligarh 202002, India E-mail address: mmt13mka@amu.ac.in

Salahuddin: Department of Mathematics, Aligarh Muslim University, Aligarh 202002, India E-mail address: salahuddin12@mailcity.com 


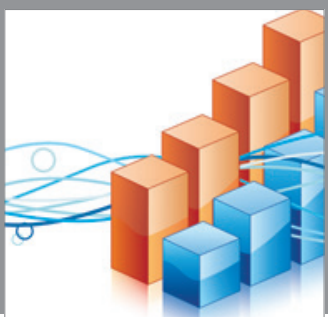

Advances in

Operations Research

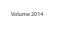

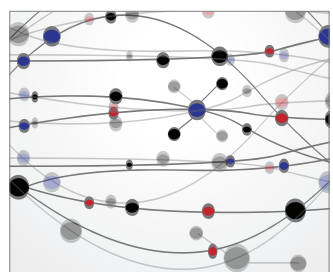

\section{The Scientific} World Journal
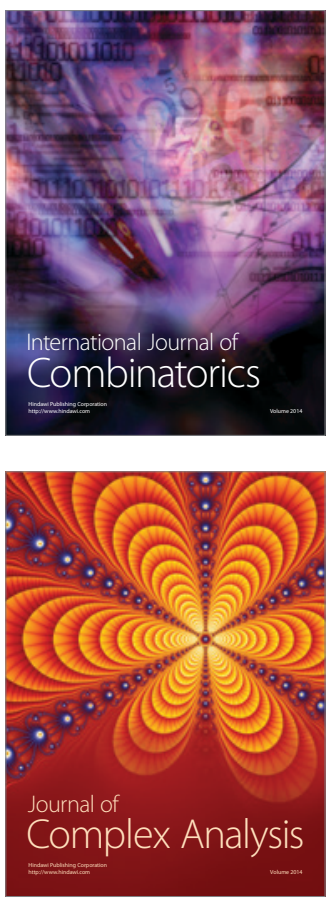

International Journal of

Mathematics and

Mathematical

Sciences
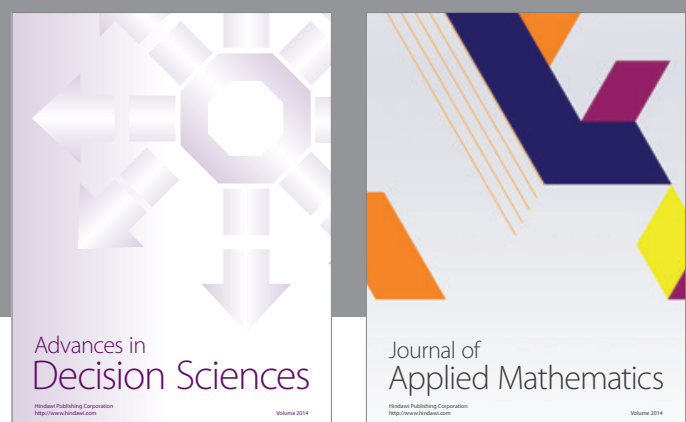

Journal of

Applied Mathematics
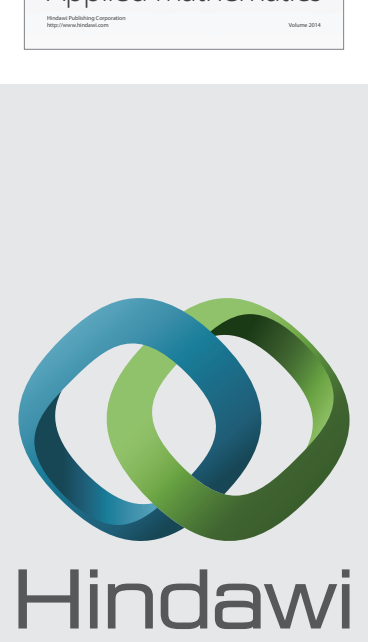

Submit your manuscripts at http://www.hindawi.com
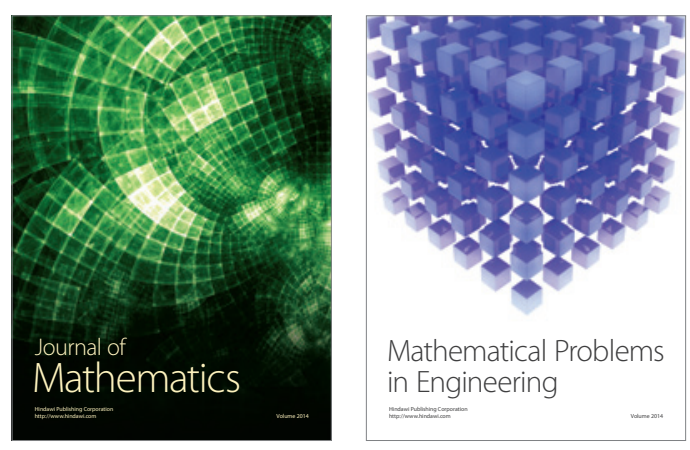

Mathematical Problems in Engineering
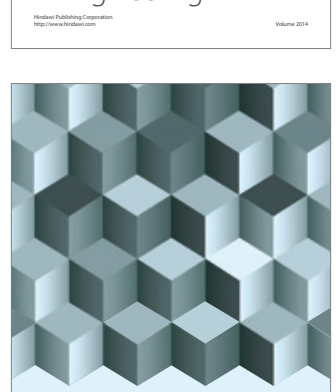

Journal of

Function Spaces
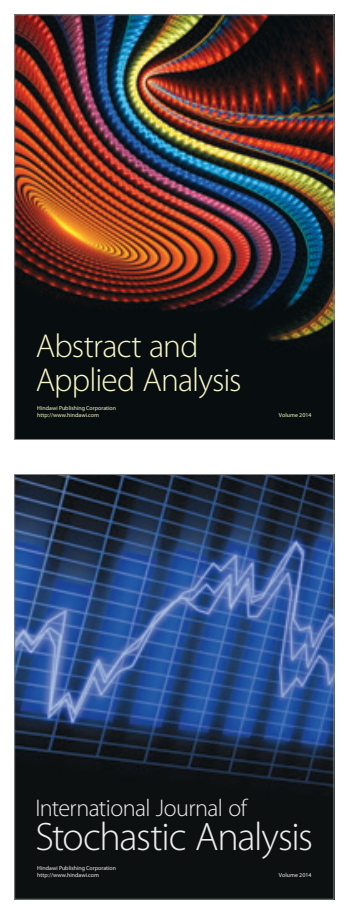

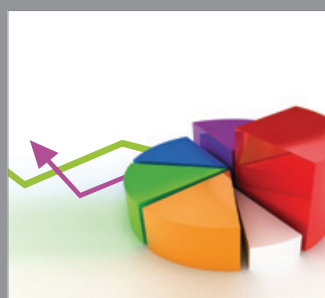

ournal of

Probability and Statistics

Promensencen
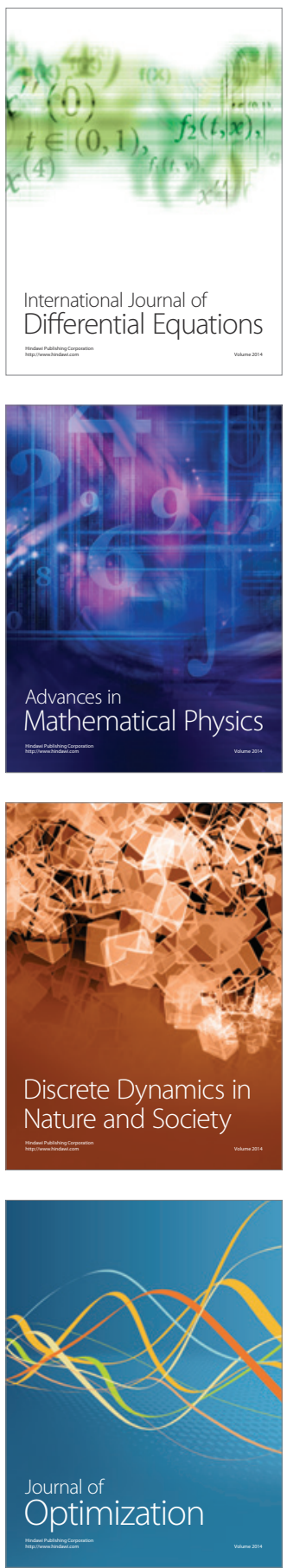